\title{
Carcinoma de Lengua Móvil.
}

\section{Mobile Tongue Carcinoma.}

\author{
José Ramón Díaz Martínez 1*iD, Jorge Damian Selgas Leite Velho1, \\ Juliette Massip Nicot1.
}

1. Instituto Nacional de Oncologia y Radiobiologia. La Habana. Cuba.

\section{*Correspondencia:}

jrdiaz@infomed.sld.cu

Teléfono [593] 032498288

Conflicto de intereses: Los autores declaran no tener conflictos de intereses.

Fondos: Ver la página 58

Recibido: 1 Enero 2018

Aceptado: 11 Abril 2018

Publicado: 30 Abril 2018

\section{Membrete bibliográfico:}

Díaz J, Selgas J, Massip J. Carninoma de lengua Móvil. Rev. Oncol. Ecu 2018;28(1):50-61.

DOI: https://doi.org/10.33821/247

Copyright Díaz, et al. Este artículo es distribuido bajo los términos de Creative Commons Attribution License, el cual permite el uso y redistribución citando la fuente y al autor original.

\section{Resumen}

Introducción: El Carcinoma de lengua móvil es uno de los tumores malignos más frecuentes de la cavidad bucal. Generalmente, los pacientes son diagnosticados después de los 40 años de edad. La detección temprana de estas lesiones, evita su desarrollo hacia fases avanzadas de la enfermedad, que oscurecen su pronóstico, por medio de una atención rápida y adecuada, biopsia oportuna, y tratamiento precoz, evitando que los pacientes sean mutilados, sufran o mueran por esta causa. El objetivo del presente estudio es reportar un grupo de pacientes con esta neoplasia, su tratamiento y supervivencia.

Métodos: En el presente estudio descriptivo, longitudinal y retrospectivo fueron evaluados los pacientes con diagnóstico de carcinoma de lengua móvil, tratados en el Instituto Nacional de Oncología de la Habana, entre los años 2007 y 2011. Se reporta edad, tabaquismo, alcoholismo, estadiaje, tipo de tratamiento y supervivencia.

Resultados: Se registraron 68 casos, se observó un predominio del grupo de edad entre los años 5569 , con el $41.18 \%$ de los pacientes, siendo el sexo predominante el masculino, con un $79.41 \%$ del total. En la clasificación TNM, se observó que predominó de los tumores estadiados como T3 con el 35.29 $\%$, seguidos de los T2 con el $30.88 \%$, la invasión ganglionar estuvo más representada por la categoría NO con el $58.82 \%$ de los casos. El $64.71 \%$ de los pacientes estaban vivos a los 60 meses.

Conclusión: El carcinoma de lengua móvil fue más frecuente en las edades entre 55-69 años y en el sexo masculino, más del $50 \%$ de los pacientes practicaban hábitos tóxicos, hubo un predominio de etapas avanzadas, la cirugía con adyuvancia postoperatoria fue la terapéutica más común.

Palabras Claves: LENGUA, GLOSECTOMÍA, NEOPLASIAS DE LA LENGUA.

DOI: $10.33821 / 247$

\section{Abstract}

Introduction: Carcinoma of the mobile tongue is one of the most frequent malignant tumors of the oral cavity. Generally, patients are diagnosed after 40 years of age. The early detection of these lesions prevents their development towards advanced stages of the disease, which obscure their prognosis, through rapid and adequate care, timely biopsy, and early treatment, preventing patients from being mutilated, suffering or dying from it cause. The objective of the present study is to report a group of patients with this neoplasia, its treatment and survival. 
Methods: In the present descriptive, longitudinal and retrospective study, patients diagnosed with mobile tongue carcinoma, treated at the National Institute of Oncology of Havana, between 2007 and 2011, were evaluated. Age, smoking, alcoholism, staging are reported, type of treatment and survival.

Results: There were 68 cases, a predominance of the age group between the years 55-69 was observed, with $41.18 \%$ of the patients, being the predominant sex the masculine, with $79.41 \%$ of the total. In the TNM classification, it was observed that tumors predominated as T3 with $35.29 \%$, followed by $\mathrm{T} 2$ with $30.88 \%$, the lymph node invasion was more represented by the N0 category with $58.82 \%$ of the cases. $64.71 \%$ of the patients were alive at 60 months.

Conclusion: The mobile tongue carcinoma was more frequent in the ages between 55-69 years and in the male sex, more than $50 \%$ of the patients practiced toxic habits, there was a predominance of advanced stages, the surgery with postoperative adjuvant was the therapeutic more common.

Keywords: TONGUE, GLOSECTOMY, NEOPLASIAS OF THE TONGUE.

DOI: $10.33821 / 247$

\section{Introducción}

Los cánceres de la lengua móvil, son las neoplasias más comunes de la cavidad oral. En EEUU, en los casos diagnosticados de cáncer de lengua desde 2002 al 2006, se observó una incidencia de 2.8 por 100 mil habitantes/año, comparando con la incidencia entre los años 1975 y 1999 de 0.4, y entre 1999 y 2006 de 2.0 se ha incrementado [1, 2]. En Europa, según datos de la IARC (Internacional Agency for Research on Cancer) la incidencia del cáncer de lengua (incluyendo base) entre los hombres oscila entre 0.8/100 mil hombres en Polonia hasta un máximo de 8/100 mil hombres en el Bajo Rin Francés. En España, el reporte varía desde 2.1 en Albacete, hasta 5.2/ 100 mil en Asturias [3]. En Cuba en el anuario estadístico del 2012 se reportaron 96 casos en el sexo masculino para una tasa cruda de 1.7 y 31 casos, en el sexo femenino para una tasa cruda de 0.6 , lo que establece una proporción de 3-1 respectivamente [4].

La etiología del carcinoma de lengua, se ha establecido la hipótesis de que las influencias ambientales contribuyen al desarrollo del mismo, éstas incluyen fumar tabaco en cigarrillos, mascar tabaco, la inhalación de humo por los fumadores pasivos, el uso de nuez de betel y el consumo de alcohol [5-8], hay otros factores de riesgo que aparecen en la literatura médica como son el bajo nivel de vida o algunas enfermedades intercurrentes como antiguamente fue la sífilis y hoy el SIDA. Las infecciones virales, como las del virus del papiloma humano también constituyen un factor asociado a la aparición de estas lesiones, generalmente el serotipo 16 [5]. El carcinoma en la boca, tradicionalmente se desarrolla después de décadas de abuso de alcohol y tabaco, también hay otros factores de riesgo como la infección por el virus de papiloma humano (VPH) [9], así como otras infecciones virales. El tabaquismo y el alcoholismo son los dos factores más importantes, esta asociación ha sido muy clara desde hace varios años [10], se estima que entre 85 y 90 $\%$ de los casos con cáncer de las vías aerodigestivas superiores se explican por la exposición al tabaco y el riesgo es proporcional a la intensidad de la exposición; así los fumadores intensos tienen un riesgo superior respecto a los fumadores ocasionales [11]. 
De acuerdo con reportes de carcinoma de cavidad oral, estos se presentan con mayor frecuencia entre la quinta y séptima década de vida con una media de 64 años $[12,13]$.

A pesar de que los factores de riesgo relacionados con el estilo de vida juegan un papel muy importante en la etiología, algunos pacientes parecen ser más susceptibles debido a un rasgo heredado en su dificultad para metabolizar carcinógenos o procarcinógenos, la sobre expresión de los oncogenes y valores de factores pronostico como el receptor del factor de crecimiento epidérmico (EGF-R) y su ligando, el factor de crecimiento transformador alfa (TGFa) está relacionado en el desarrollo de células tumorales: los altos valores de ambos, ligando y receptor, en tumores y en mucosa normal de pacientes con cáncer de cabeza y cuello indican que las influencias ambientales, tales como el uso del tabaco y el alcohol, conducen a la sobre regulación de la producción de factores de crecimiento y expresión del receptor, lo que puede participar en el desarrollo tumoral [14].

El objetivo del presente estudio es describir los factores de riesgo, el tratamiento recibido y la supervivencia de un grupo de pacientes con carcinoma de lengua móvil que asistieron al tratamiento en un centro oncológico único.

\section{Materiales y Métodos}

En el presente estudio descriptivo, retrospectivo, se evaluaron los expedientes clínicos de los pacientes con diagnóstico inicial de carcinoma de de lengua móvil, tratados en el Instituto Nacional de Oncología de La Habana-Cuba, en el período de enero de 2007 a diciembre de 2011. Las variables evaluadas fueron la edad, el sexo, la ubicación topográfica y la clasificación clínica de la lesión, TNM, los diferentes tratamientos utilizados y sus resultados, así como el estado en su última visita al Instituto. Se excluyeron casos en los cuales la información no estuvo completa así como los casos sin diagnóstico histopatológico. El universo constituyeron todos los casos posibles en el período de estudio. El análisis estadístico realizado fue descriptivo para las variables como edad, tipo de tratamiento y estadío. Se utilizó el método de Kaplan Meier para medir la supervivencia.

\section{Resultados}

Ingresaron al estudio 68 casos de pacientes con carcinoma de lengua móvil. Se observó un predominio del grupo de edad entre los $55-69$ años, con el $41.18 \%$ de los pacientes, siendo el sexo más frecuente el masculino, representado por el $79.41 \%$ de la población, con una proporción de 4:1 (Tabla 1). Los factores de riesgo de tabaquismo y alcoholismo juntos fue más representativo con $33.82 \%$ de los casos, seguido de los pacientes que solamente fumaban con el $27.95 \%$ de los mismos (Tabla 2).

Se observó que predominaron los tumores estadiados como T3 con el $35.29 \%$, seguidos de los T2 con el $30.88 \%$, la invasión ganglionar estuvo más representada por la categoría NO con el $58.82 \%$ de los casos, no se evidenció existencia de metástasis a distancia, en el 
momento del diagnóstico en el $100 \%$ de los pacientes. La etapa clínica predominante fue la III, con el $30.88 \%$ de los casos, a expensas del predominio del tamaño tumoral (T3), invasión ganglionar (N1), seguida de la etapa IVa, con el $27.94 \%$ de los casos, justificado fundamentalmente por existir 16 pacientes con N2 (Tabla 3 ).

Tabla 1. Carcinoma de lengua móvil. Descripción de los pacientes con según grupo de edad y sexo.

\begin{tabular}{|c|c|c|c|}
\hline \multirow{2}{*}{$\begin{array}{c}\text { Grupo de } \\
\text { Edad }\end{array}$} & \multicolumn{3}{|c|}{ Sexo } \\
\hline & $\begin{array}{c}\text { Masculino } \\
n=54(79.41 \%)\end{array}$ & $\begin{array}{c}\text { Femenino } \\
n=14(20.59 \%)\end{array}$ & $\begin{array}{c}\text { Total } \\
n=68(100 \%)\end{array}$ \\
\hline 25- 39 & $2(3.7 \%)$ & $3(21.43 \%)$ & $5(7.35 \%)$ \\
\hline $40-54$ & $19(35.19 \%)$ & $3(21.43 \%)$ & $22(32.35 \%)$ \\
\hline $55-69$ & $23(42.59 \%)$ & $5(35.71 \%)$ & $28(41.18 \%)$ \\
\hline 70- 84 & $10(18.52 \%)$ & $2(14.29 \%)$ & $12(17.65 \%)$ \\
\hline 85 y más & - & $1(7.14 \%)$ & $1(1.47 \%)$ \\
\hline
\end{tabular}

Tabla 2. Hábitos tóxicos referidos por los pacientes.

\begin{tabular}{|l|c|c|}
\hline Hábitos tóxicos & $\mathbf{n = 6 8}$ & $\%$ \\
\hline Tabaquismo & 19 & 27.95 \\
\hline Alcoholismo & 5 & 7.35 \\
\hline Tabaquismo y alcoholismo & 23 & 33.82 \\
\hline Otros & 5 & 7.35 \\
\hline Ninguno & 16 & 23.53 \\
\hline
\end{tabular}

Tabla 3. Estadiaje clínico

\begin{tabular}{|l|c|c|l|c|c|l|c|c|l|c|c|}
\hline T. & No. & $\%$ & N. & No. & $\%$ & M. & No. & $\%$ & Etapa & No. & $\%$ \\
\hline Tis & 2 & 2.94 & Nx & - & - & M0 & 68 & 100 & 0 & 2 & 2.94 \\
\hline T0 & - & - & N0 & 40 & 58.82 & & & & I & 8 & 11.77 \\
\hline T1 & 9 & 13.24 & N1 & 11 & 16.18 & & & & II & 15 & 22.06 \\
\hline T2 & 21 & 30.88 & N2a & 5 & 7.35 & M1 & - & - & III & 21 & 30.88 \\
\hline T3 & 24 & 35.29 & N2b & 10 & 14.71 & & & & IVa & 19 & 27.94 \\
\hline T4a & 9 & 13.24 & N2c & 1 & 1.47 & & & & IVb & 3 & 4.41 \\
\hline T4b & 3 & 4.41 & N3 & 1 & 1.47 & & & & IVc & - & - \\
\hline
\end{tabular}

El tratamiento con cirugía seguido de quimio-radioterapia, fue el más usado, en el $33.82 \%$ de los pacientes, seguido de la cirugía como tratamiento único en el $29.41 \%$ de los casos, justificado por que hubo 32 con lesiones menores de $4 \mathrm{~cm}$ en el momento de diagnóstico y en concordancia con los protocolos de tratamiento (Tabla 4). 
Tabla 4. Modalidades de tratamiento aplicadas.

\begin{tabular}{|l|c|c|}
\hline Modalidades de tratamiento. & \multicolumn{2}{|c|}{ Pacientes } \\
\cline { 2 - 3 } & No. & $\%$ \\
\hline Cirugía & 20 & 29.41 \\
\hline Cirugía más Radioterapia adyuvante & 17 & 25.00 \\
\hline Cirugía y Radioterapia - quimioterapia adyuvante & 23 & 33.82 \\
\hline Radioterapia-Quimioterapia 1ra opción & 7 & 10.30 \\
\hline Radioterapia & 1 & 1.47 \\
\hline Total & 68 & 100 \\
\hline
\end{tabular}

Tabla 5. Técnica quirúrgica utilizada, según clasificación T.

\begin{tabular}{|c|c|c|c|c|c|c|c|c|c|c|c|c|c|c|}
\hline \multirow{3}{*}{\begin{tabular}{|l|} 
Técnica \\
Quirúrgica
\end{tabular}} & \multicolumn{14}{|c|}{ Clasificación clínica ( T). } \\
\hline & \multicolumn{2}{|c|}{ Tis } & \multicolumn{2}{|c|}{$\mathrm{T1}$} & \multicolumn{2}{|c|}{ T2 } & \multicolumn{2}{|c|}{ T3 } & \multicolumn{2}{|c|}{ T4a } & \multicolumn{2}{|c|}{ T4b } & \multicolumn{2}{|c|}{ Total } \\
\hline & No & $\%$ & No & $\%$ & No & $\%$ & No & $\%$ & No & $\%$ & No & $\%$ & No & $\%$ \\
\hline $\begin{array}{l}\text { Glosectomía } \\
\text { parcial }\end{array}$ & 2 & 100 & 8 & 88.89 & 15 & 71.43 & 8 & 33.33 & - & - & - & - & 33 & 48.53 \\
\hline Hemiglosectomía & - & - & - & - & 1 & 4.76 & 4 & 16.67 & - & 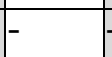 & - & - & 5 & 7.35 \\
\hline Pelviglosectomía & - & - & - & - & 4 & 19.05 & 3 & 12.50 & 2 & 22.22 & - & - & 9 & 13.24 \\
\hline Ninguna & - & - & - & - & 1 & 4.76 & 3 & 12.50 & 1 & 11.11 & 3 & 100 & 8 & 11.76 \\
\hline Otras & - & - & 1 & 11.11 & - & - & 6 & 25.00 & 6 & 66.67 & - & - & 13 & 19.12 \\
\hline Total & 2 & 2.94 & & 13.24 & 21 & 30.88 & 24 & 35.29 & 9 & 13,24 & 3 & 4.41 & 68 & 100 \\
\hline
\end{tabular}

Al distribuir la técnica quirúrgica local utilizada, se puede observar que en los estadíos iníciales Tis, T1 y T2 predominaron las glosectomías parciales con el $100 \%, 88.89 \%$ y el $71.43 \%$ respectivamente, llamó la atención que en 8 pacientes estudiados como T3, también le fue realizada esta técnica. En etapas avanzadas se realizaron predominantemente otras técnicas, lo cual quiere decir que involucraron el manejo de la mandíbula en los T3, en un $25 \%$; y en los T4a en el $66.67 \%$ de los casos. A los 3 pacientes estudiados como T4b no se les realizó ninguna tipo de tratamiento quirúrgico (Tabla 5).

Se determinó la supervivencia en función de la etapa clínica, (Figura 1). En cuanto a la presencia de la enfermedad a los 60 meses, el $100 \%$ de los pacientes en etapa 0 y I se encontraron controlados, mientras que, en etapas avanzadas, III y IVa del total de pacientes controlados, el $57.89 \%$ y 53.85 presentaron recaída de la enfermedad; el $63.16 \%$ de los pacientes se encontraron controlados. Se determinó la supervivencia libre de enfermedad. (Figuras 1 y 2).

No hubo diferencias significativas entre las diferentes etapas clínicas en los pacientes según recaída de la enfermedad $(P=0.225)$. A pesar de que en las etapas 0 y l el $100 \%$ los pacientes se mantuvieron controlados, mientras que en las etapas II, III y IV se mantuvieron libres de enfermedad un promedio de $28,48,25,20$ y 15,61 meses respectivamente. 
Al identificar el estado de los pacientes a los 60 meses se observó que en las etapas tempranas, 0 y I el $100 \%$ estaban vivos y en la II el $86.67 \%$, mientras que en la enfermedad muy avanzada, etapa IVa y IVb, el $57.90 \%$ y el $100 \%$ respectivamente se encontraban fallecidos. Se constató que el 64.71 \% de los pacientes se encontraban vivos. Se determinó la supervivencia global, siendo significativa la relación entre estas dos variables (Figuras 3 y 4).

En los pacientes en etapa clínica 0 y I no hubo fallecidos al término del estudio. Los pacientes en etapa II tuvieron una supervivencia de $114 \pm 6$ meses, en los de etapa III fue de $86 \pm 10$ meses mientras que los que tenían la etapa más avanzada (IV) sobrevivieron 56 \pm 11 meses como promedio. Las diferencias resultaron estadísticamente significativas $(P<0.001)$.

Figura 1. Tiempo libre de enfermedad según etapa clínica.

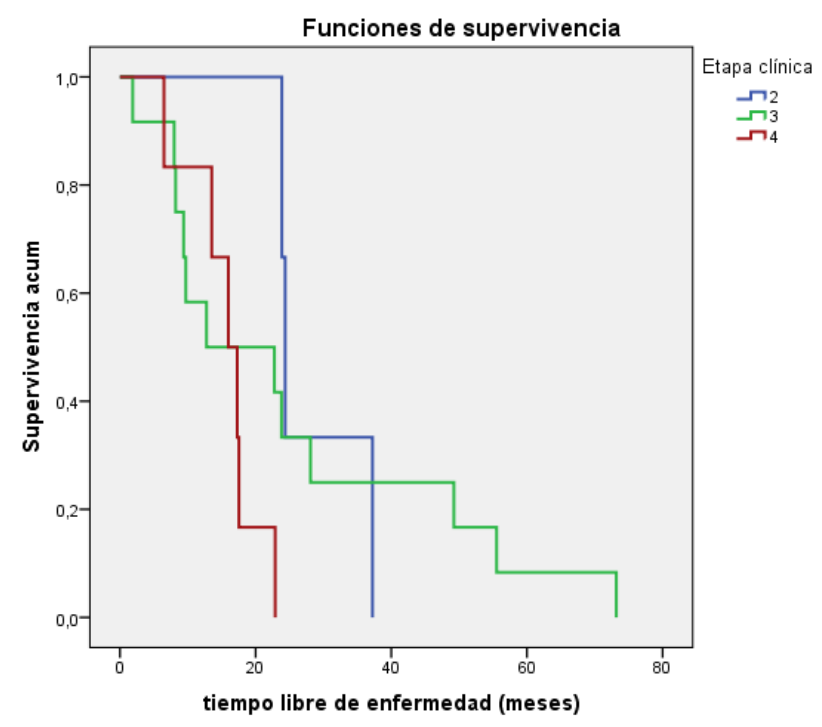

Figura 2. Tiempo libre de enfermedad global.

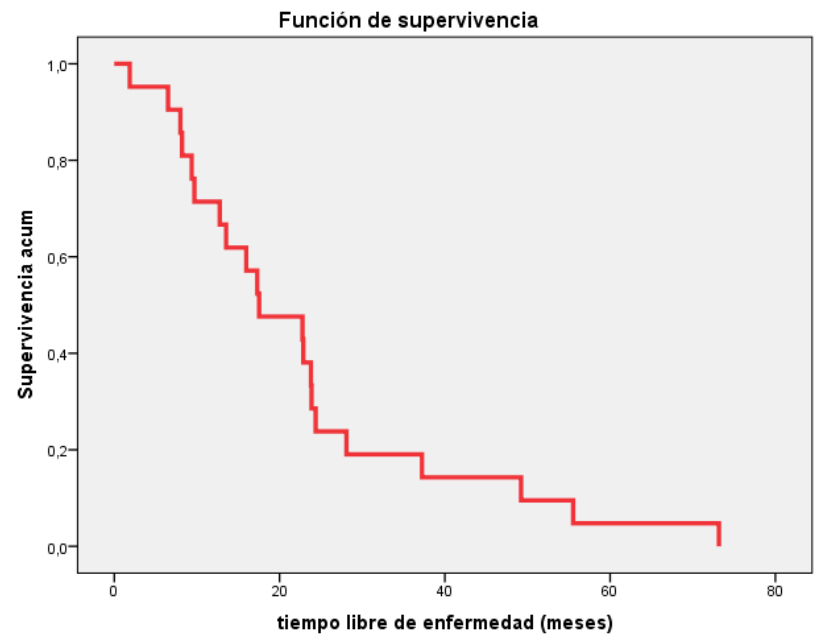


Figura 3. Supervivencia según etapa clínica.

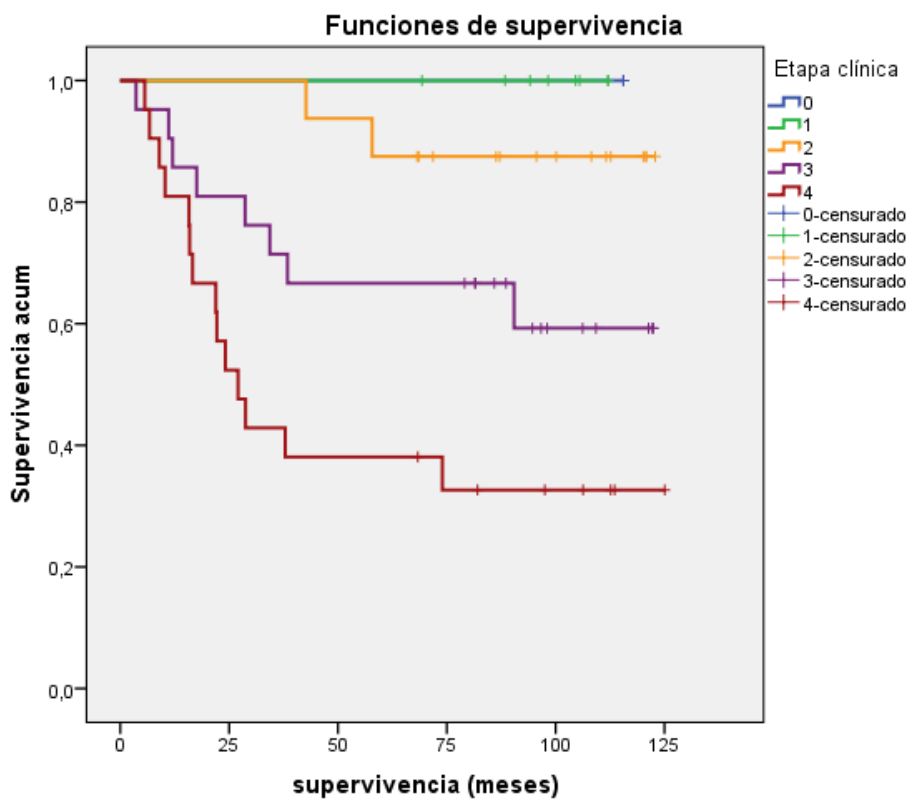

Figura 4. Supervivencia global.

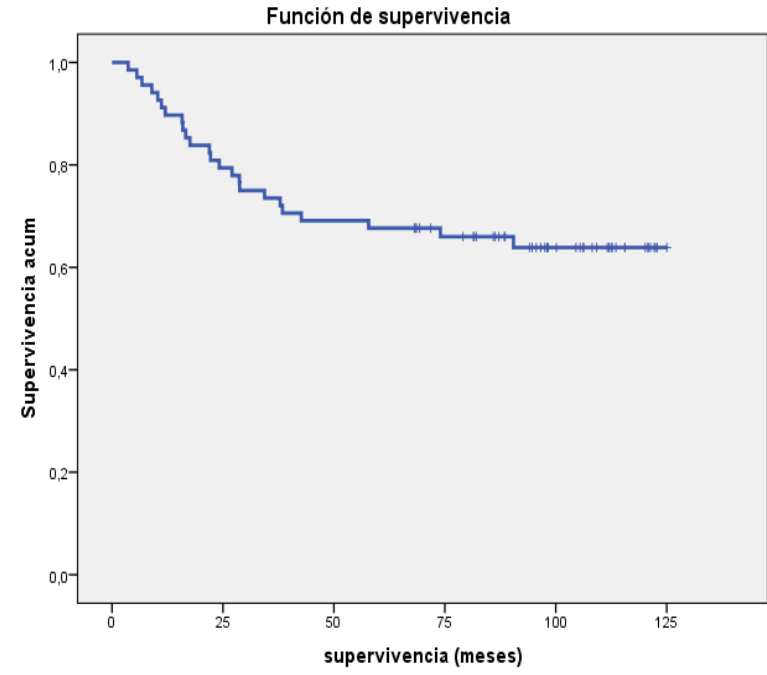

\section{Discusión}

En la presente casuística, en el carcinoma de lengua móvil, la combinación de tabaquismo y alcoholismo fue lo más representativo con $33.82 \%$. En un estudio que incluyó 173 pacientes con carcinoma de células escamosas y 176 controles sanos, cuando se ajustó el riesgo por tabaquismo y alcoholismo se encontró que el riesgo para padecer cáncer era 2,6 asociado al consumo de marihuana en forma proporcional a la cantidad [15]. Este 
estudio no demostró la presencia o ausencia del VPH. En un estudio previo [16] de 105 pacientes se demostró que ninguno de ellos fueron positivos para $\mathrm{VPH}$, lo que sugiere que la incidencia del VPH en el cáncer de la parte oral de la lengua es baja y es poco probable que este virus juegue un papel importante en la etiología, patogenia y resultados clínicos [29] y adicionalmente hay estudios [18], que sugieren que la presencia de VPH es un factor pronóstico favorable en los cánceres de base de lengua. El promedio de edad reportado es de 60 y 65 años de edad $[19,20]$, aunque en estos últimos 10 años se expone un incremento de su incidencia en la población joven [21, 22]. En el presente reporte la edad prevalente fue 55- 69 años, aunque se evidenció incremento en la población más joven, lo cual pudiera estar relacionado con los estilos de vida no saludables.

Esta enfermedad afecta más al sexo masculino, debido, posiblemente, a que es este grupo el que más se expone a prácticas nocivas o hábitos tóxicos que condicionan el cáncer bucal, no obstante, los resultados encontrados, no difieren con los de otras bibliografías consultadas [22,23] en las cuales los hombres padecen 2 ó 3 veces más el cáncer de lengua con respecto a las mujeres, resultados que concuerdan con los encontrados en este estudio, en donde resalta el sexo masculino. En la presente investigación existe coincidencia con un estudios previos [24, 25], en lo que se reportaron $69-78.9 \%$ de fumadores y un $20-54 \%$ de consumidores de bebidas alcohólicas.

En estudio previos [22] en una muestra de 94 pacientes, obtuvieron que más de la mitad de los casos correspondía a las etapas tres y cuatro, al igual que otro estudio [19], en la que en una serie de 187 pacientes que el $56.2 \%$ correspondía a la etapa cuatro estos datos coinciden con el presente reporte, evidenciándose una tendencia al diagnóstico tardío, lo cual repercute negativamente en la sobrevida y calidad de esta.

En un estudio previo [26], se reportaron que el tratamiento con radioterapia junto a la quimioterapia en los protocolos de preservación del órgano, se aplicó en un $44.4 \%$ de los casos. En la presente investigación coinciden las modalidades terapéuticas más utilizadas, con las referidas por los diferentes autores antes mencionados,

Los tumores de la lengua móvil tienen una alta incidencia de metástasis ganglionares regionales, entre $15-75 \%$ de los pacientes dependiendo del estadio inicial del tumor primario. En estadios precoces, la incidencia de metástasis oculta oscila entre 20-40\% según las distintas series quirúrgicas. Entre un 3-5\% debutan con afectación ganglionar bilateral y un 15-20\% presenta metástasis ganglionares contralaterales [27].

Se ha identificado un escaso índice de supervivencia global a los 5 años, inferior al $50 \%$ debido a su diagnóstico tardío y a la diseminación metastásica cervical incontrolada, especialmente si consideramos el hecho de que la evaluación clínica del mismo, incluso con el apoyo de técnicas de imagen, permite únicamente una detección del $68 \%$ de los casos, con invasión ganglionar [28, 29].

La supervivencia está en evidente relación con el tamaño del tumor, siendo del 60-70\% para estadios I- II, pero cayendo hasta el 30-40\% para los estadios III- IV [30].

Algunos estudios consideran la edad al momento del diagnóstico como posible factor pronóstico, estimando una sobrevida libre de enfermedad del $48 \%$ para pacientes con edad 
menor de 45 años y $54 \%$ en mayores de 46 años, no encontrándose diferencia significativa entre ambos grupos, en nuestro estudio. Se han reportado un $38 \%$ de recurrencia, siendo mayor la recurrencia local que la recurrencia regional [29], en la presente serie se encontró resultados similares, lo que nos lleva a concluir que el manejo adecuado de los tumores de lengua comienza con la prevención de sus factores de riesgo, diagnóstico precoz, tratamiento oportuno y un seguimiento adecuado que al final se traducirían en alto control de la enfermedad, con óptima calidad de vida.

\section{Conclusiones}

El carcinoma de lengua móvil en la muestra estudiada, se comporta igual a lo descrito internacionalmente, predominando en edades avanzadas, en el grupo etario 55-69 años de edad y en el hombre, de igual forma en cuanto a la influencia de hábitos tóxicos, predominaron los pacientes que fumaban e ingerían bebidas alcohólicas. A pesar de los programas establecidos en Cuba, para el diagnóstico precoz siguen llegando los pacientes en etapas avanzadas, predominando la etapa III, seguida de la etapa IV. Los tratamientos utilizados cumplen con los protocolos nacionales e internacionales y las curvas de supervivencias están por encima de la media de supervivencia.

\section{Agradecimientos}

Reconocemos a las personas que participaron indirectamente en el estudio tales como el personal técnico, familiares y visitantes del Instituto Nacional de Oncología y Radiobiología. La Habana-Cuba.

\section{Información adicional}

Nota del Editor

La Revista Oncología Ecu permanece neutral con respecto a los reclamos jurisdiccionales en mapas publicados y afiliaciones institucionales.

\section{Abreviaturas}

IARC: Internacional Agency for Research on Cancer.

\section{Archivos Adicionales}

Ninguno declarado por los autores.

\section{Fondos}

Los fondos de la investigación fueron propios de los autores del presente artículo. 
Disponibilidad de datos y materiales

Existe la disponibilidad de datos bajo solicitud al autor de correspondencia. No se reportan otros materiales.

\section{Contribuciones de los autores}

JRDM, JDSLV realizaron la idea de investigación, revisión bibliográfica. JDSLV y JMN, recolección de datos, escritura del artículo. JRDM realizó el análisis crítico del artículo. Todos los autores leyeron y aprobaron la versión final del artículo.

Aprobación de ética y consentimiento para participar

No aplica a este estudio.

\section{Consentimiento para publicación}

No aplica.

\section{Información de los autores}

José Ramón Díaz Martínez, Médico Especialista de II grado en Oncología. Investigador Auxiliar. Cirujano de Cabeza y Cuello. Instituto Nacional de Oncología y Radiobiología. La Habana. Cuba.

Jorge Damian Selgas Leite Velho. Especialista de I grado en Cirugía Maxilofacial. Instituto Nacional de Oncología y Radiobiología. La Habana. Cuba.

Juliette Massip Nicot. Especialista de 1rogrado en Bioestadística. Instituto Nacional de Oncología y Radiobiología. La Habana. Cuba.

\section{Revisiones por pares \\ Acceda a la revisión de pares académicos en el siguiente enlace: https://publons.com/review/3811144/}

\section{Referencias}

1. García Kass Al, Domínguez Gordillo AA, García Núñez JA, Revisión y puesta al día en cáncer de lengua, Avances en odonto estomatología. 2013;29(5):15. SU: goo.gl/fkecDq 
Abreviaturas en la referencias

DOI: Digital Object

Identifier

PMID: PubMed Identifier

SU: Short URL
2. Rana $M$, Iqbal $A$, Warraich $R$, Ruecker $M$, Eckardt $A M$, GellrichNC, et al. Modern surgical management of tongue carcinoma-A clinical retrospective research over a 12 years period. Head Neck Oncol. 2011;3:43. DOI: $10.1186 / 1758-3284-3-43$

3. Carreras-Torras C, Gay-Escoda C. Techniques for early diagnosisof oral squamous cell carcinoma: Systematic review. Med OralPatol Oral Cir Bucal. 2015; 20(3):305-15.2. DOI: 10.4317/medoral.20347

4. Col. Autores. Registro Nacional de Cáncer. Incidencia de cáncer según sexo y localización, número de casos, tasas y ajustadas, Cuba 2012. Revista cubana de ORL 2012;1-28. DOI:10.1186/1471-2407-14$\underline{535}$

5. Guerrero LC, Muñoz HJ, Sáenz de Miera JB, Pérez NR, Reynales SL. Impacto del consumo nocivo de alcohol en accidentes y enfermedades crónicas en México. Salud Publica Mex. 2013;55(Sup2):28288. SU: goo.gl/z1gCkL

6. Ordoñez D, Aragón N, García LS, Collazos P, Bravo LE. Cáncer oral en Santiago de Cali, Colombia: análisis poblacional de la tendencia de incidencia y mortalidad. Salud Pública de México.2014; 56(5):465-72. SU: goo.gl/jBkDjG

7. Parks KA, Collins RL, Derrick JL. The influence of marijuana and alcohol use on condom use behavior: findings from a sample of young adult female bar drinkers. Psychol Addict Behav 2012;26:888-94. DOI: $\underline{10.1037 / a 0028166}$

8. Guerrero LC, Muñoz HJ, Sáenz de Miera JB, Reynales SL. Consumo de tabaco, mortalidad y política fiscal en México. Salud Pública Mex. 2013;55(Sup 2):276-81. SU: goo.gl/DKFpFc

9. González-Ramírez I, Irigoyen-Camacho ME, Ramírez-Amador V, Lizano-Soberón M, Carrillo-García A García-Carrancá A et al. Association between age and high-risk human papilloma virus in Mexican oral cancer patients. Oral Dis. 2013;19(8):796-804. DOI: 10.1111/odi.12071

10. Momares B, Contreras G, Martínez B, Ávalos N, Carmona L. Sobrevida en carcinoma espinocelular de mucosa oral: análisis de 161 pacientes. Rev Chil Cir 2014;66(1):568-76. DOl:10.4067/s071840262014000600010

11. Sun $Q$, Fang $Q$, Guo $S$. A comparison of oral squamous cell carcinoma between young and old patients in a single medical center in China. Int J Clin Exp Med. 2015;8(8):12418-423. PMID: 26550153

12. Winzer KJ, Bellach J, Hufnagl P. Long-term analysis to objectify the tumour grading by means of automated microscopic image analysis of the nucleolar organizer regions (AgNORs) in the case of breast carcinoma. Diagn Pathol. 2013;8:56. DOI: 10.1186/1746-1596-8-56

13. Martínez-Cortez I, Martínez-Mejía V, Amezcua-Rosas G, et al. Diagnóstico tardío de carcinoma escamo celular en boca, reporte de caso. Int J Odontostomt. 2011;5(3):240-44. DOI: 10.4067/s0718$381 \times 2011000300006$

14. Carrillo RJ, Simón NE, Gil Romero M, Rodríguez FM. Cáncer oral en México. Revisión bibliográfica y presentación de caso clínico. Rev AMCBM. 2011;7(3):104-08. SU: goo.gl/nfwfQJ

15. Moral de la Rubia J. Homosexualidad en la juventud mexicana y su distribución geográfica. Rev Papeles de Poblacion. 2011;17(67):115-19. SU: goo.gl/o8YrBv

16. Carvalho de Melo A, Pedreira Ramalho L, Ferreira Ribeiro C, Diniz da ROSA M. Informação e Comportamento Preventivo de Pacientes do Programa de Saúde da Família de Aracaju a Respeito de Câncer Bucal. Pesquisa Brasileira Em Odontopediatria E Clinica Integrada. 2012;12(3):101-112. DOI: 10.4034/PBOCl.2012.123.12

17. Lingen MW, Xiao W, Schmidt A, Jiang B, Pickard R, Kreinbrink $P$, et al. Low etiologic fraction for high-risk human papilloma virus in oral cavity squamous cell carcinomas. Oral Oncol 2012;49:1-8. DOI: 10.1016/j.oraloncology.2012.07.002

18. Dissanayaka WL, Pitiyage G, Kumarasiri PV, Liyanage RL, Dias KD, Tilakaratne WM. Clinical and histopathologic parameters in survival of oral squamous cell carcinoma. Oral Surg Oral Med Oral Pathol Oral Radiol. 2012;113:518-25. DOI: 10.1016/j.0000.2011.11.001 
19. Rivera C, Gonzalez-Arriagada WA, Loyola-Brambilla M, de Almeida OP, Coletta RD, Venegas B. Clinicopathological and immunohistochemical evaluation of oral and oropharyngeal squamous cell carcinoma in Chilean population. Int $\mathrm{J}$ Clin Exp Pathol. 2014;7: 5968-77. DOI: 10.1016/j.0000.2011.11.001

20. Doncel Pérez C, Méndez Calunga M, Betancourt Dieste H, Castillo Castillo A. Conocimientos sobre el cáncer bucal en pacientes de Estomatología. Rev Cub Med Mil. Marzo 2014; 43(1):48-60. SU: goo.gl/Mc73Rs

21. Alexei Pérez y col. Revista de ciencias médicas de Pinar del Río. Nov.- Dic 20016. Morbilidad del carcinoma epidermoide de lengua. Pinar del Río 2000-2016. 2016;20(6):690-697. SU: goo.gl/1dtSuS

22. Jadhav KB, Gupta N. Clinicopathological prognostic implicators of oral squamous cell carcinoma: Need to understand and revise. N Am J Med Sci. 2013;5:671-79. DOI: 10.4103/1947-2714.123239

23. Miranda Tarragó JD, Gispert Abreu EA. Intersectorialidad de salud en la prevención del cáncer bucal. Rev cubana Estomatol. 2012;49(4):14-22. SU: goo.gl/RDqwEv

24. Dragomir LP, Simionescu C, Margaritescu A, Stepan IM, Dragomir, MR, Popescu. "P53, p16 and Ki67 immunoexpression in oral squamous carcinomas." Rom J Morphol Embryol 2012;53(1):89-93. SU: goo.gl/LPAedu

25. Durr ML, Zante D, Li EJ, Kezirian SJ, Wang. Oral Tongue Squamous Cell Carcinoma in Never-Smokers: Analysis of Clinicopathologic Characteristics and Survival. Otolaryngol Head Neck Surg 2013. DOI: $10.1177 / 0194599813482876$

26. Momares B, Contreras G, Martínez B, Ávalos N, Carmona L. Sobrevida en carcinoma espinocelular de mucosa oral: análisis de 161 pacientes. Rev Chil Cir. 2014; 66:568-76. DOI:10.4067/s0718$\underline{40262014000600010}$

27. Koontongkaew S. The tumor microenvironment contribution to development, growth, invasion and metastasis of head and neck squamous cell carcinomas. J Cancer. 2013;4:66-83. DOI: 10.7150/jca. 5112

28. Guevara-Canales JO, Morales-Vadillo SJ, Sacsaquispe-Contreras C, Barrionuevo-Cornejo J, Montes-Gil $\mathrm{CE}$, Cava-Vergiu FA, et al. Malignant lymphoma of the oral cavity and the maxillofacial region: overall survival prognostic factors. Med Oral Patol Oral Cir Bucal 2013;18(4):619-626. DOI: $10.4317 /$ medoral. 18903

29. Hubert Low TH, Gao M, Elliott JR, Clark. Tumor classification for early oral cancer: Re-evaluate the current TNM classification. Head Neck 2015;37(2):223-8. DOI: 10.1002/hed.23581.

30. Word Health Organization. IARC. "IARC Monographs on the Evaluation of carcinogenic Risk On Humans." 2014. SU: goo.gl/FA6iq6 